BULLETIN OF THE

AMERICAN MATHEMATICAL SOCIETY

Volume 77. Number 5, September 1971

\title{
MINIMAL SURFACES WITH MOVABLE BOUNDARIES ${ }^{1}$
}

\author{
BY JOHANNES C. C. NITSCHE
}

Communicated by P. D. Lax, April 8, 1971

A general version of Plateau's problem may be formulated as follows: Determine a surface of least area which has a prescribed topological type and which is bounded by a configuration of several fixed contours, supporting surfaces, and movable threads of given lengths.

Soap laminae spanning frames with movable contours-experimentally realized by hairs or thin threads - have been described, among other places, in $[2$, p. 48], [3, p. 174], [8, p. 584], [13, pp. $7,8,20]$. As is well known, the existence of a solution hinges on certain metric-geometric conditions which must be satisfied by the bounding configuration. Otherwise the minimizing sequences on which the existence proof is based do not converge or, if they do, converge only to degenerate limit structures-disconnected surfaces of lower topological types or partly one-dimensional formations. There are interesting examples illustrating these contingencies. In a number of cases, however, existence proofs are feasible by an extension of the classical methods, leading to a solution surface whose position vector $\mathfrak{r}(u, v)$ appears as function of isothermal parameters.

Today the boundary behavior of the solution surface is fully studied for the fixed contours and is satisfactorily understood for the free boundaries, i.e., the boundary portions on supporting manifolds. Almost nothing, however, is known so far for the moveable contours. In [11] a theorem had been proved provided certain regularity assumptions dictated by intuition could be taken for granted:

TheOREM. The movable parts of the boundary must be curves of constant space curvature. On the solution surface itself they are asymptotic lines of constant geodesic curvature.

As was pointed out in [11], there is an obvious and challenging connection to the isoperimetric problem on surfaces.

It is the purpose of this announcement to report on new results concerning the regularity of the moveable boundaries which justify the intuitive assumptions mentioned above. Details and full proofs will appear elsewhere.

It will suffice to consider the concrete case of a frame which consists of a fixed rectifiable Jordan arc $\Gamma_{1}$ and a moveable arc $\Gamma_{2}$ or prescribed

AMS 1969 subject classifications. Primary 4900, 5304; Secondary 2656, 3545.

1 Supported by AFOSR 883-67. 
length $\ell_{2}$ (smaller than the length $\ell_{1}$ of $\Gamma_{1}$ ) having its end points in common with $\Gamma_{1}$.

Denote by $P$ the semidisc $\left\{u, v ; u^{2}+v^{2}<1, v>0\right\}$ and let $S=\{\mathfrak{r}=\mathfrak{r}(u, v) ;(u, v) \in \bar{P}\}$ be a surface of least area-more precisely, a surface minimizing the value of Dirichlet's integral $D[\mathfrak{r}]$ $=\frac{1}{2} \iint_{P}\left(\mathfrak{r}_{u}^{2}+\mathfrak{r}_{v}^{2}\right) d u d v$-bounded by this frame. It is assumed here that the position vector of $S$ possesses the following properties:

(i) $\mathfrak{r}(u, v)$ belongs to class $C^{0}(\bar{P}) \cap C^{2}(P)$ and satisfies in $P$ the relations $\Delta \mathfrak{r}=\mathfrak{b}, \mathfrak{r}_{u}^{2}=\mathfrak{r}_{v}^{2}, \mathfrak{r}_{u} \mathfrak{r}_{v}=0$.

(ii) $\mathfrak{r}(u, v)$ provides a topological mapping of the boundary portion $\partial_{1} P=\left\{u, v ; u^{2}+v^{2}=1, v \geqq 0\right\}$ onto the arc $\Gamma_{1}$.

(iii) The vector $\mathfrak{r}(u, 0)$ is of bounded variation for -1 $\leqq u \leqq 1$ and $\int_{-1}^{+1}|d \mathfrak{r}(u, 0)|=l_{2}$, i.e., the boundary portion $\partial_{2} P=\{u, v ;-1 \leqq u \leqq 1, v=0\}$ is mapped onto a rectifiable arc, the curve $\Gamma_{2}$, of length $l_{2}$.

From $\ell_{2}<\ell_{1}$ it can then be inferred that $D[\mathrm{r}]>0$.

It should be stressed once more that the existence proof which leads to a solution surface having the above properties is possible only in certain situations.

For $0<r<1$ let $P_{r}$ be the semidisc $\left\{u, v ; u^{2}+v^{2}<r^{2}, v>0\right\}$. We now state the theorem: ${ }^{2}$

TheOREM. The vector $\mathfrak{r}(u, v)$ belongs to class $C^{2, \beta}\left(\bar{P}_{r}\right)$ for every $r$, $0<r<1$. Here $\beta=\beta(r)$ is an exponent between zero and one depending on $r$. The curve $\{\mathfrak{r}=\mathfrak{r}(u, 0) ;-1<u<1\}$ is a regular $C^{2}$-curve of constant space curvature.

Denote by $\mathrm{r}^{*}(u, v)$ the harmonic vector conjugate to $\mathfrak{r}(u, v)$. From the rectifiability of the boundary of $S$ it follows that $\mathrm{r}^{*}(u, v)$ is continuous in $\vec{P}$. As a matter of fact, by a theorem of M. Tsuji [12], the vectors $\mathfrak{r}(u, v)$ and $\mathfrak{r}^{*}(u, v)$ are absolutely continuous on $\partial P$ satisfying the relations $\mathfrak{r}_{u}=\mathfrak{r}_{v}^{*}, \mathfrak{r}_{v}=-\mathfrak{r}_{u}^{*}$ almost everywhere. In particular, the derivative $\mathfrak{r}_{u}(u, 0)$ exists almost everywhere on $\partial_{2} P$ and $\ell_{2}$ $=\int_{-1}^{+1}\left|\mathfrak{r}_{u}(u, 0)\right| d u$. These facts are essential for the derivations to follow.

Consider now two vectors $\mathfrak{y}(u, v)$ and $z(u, v)$ of class $C^{1}(\bar{P})$ which vanish along $\partial_{1} P$. Introducing two small parameters $\epsilon$ and $\delta$ form the comparison surface

$$
\bar{S}=\{\mathfrak{r}=\overline{\mathfrak{r}}(u, v ; \epsilon, \delta) \equiv \mathfrak{r}(u, v)+\epsilon \mathfrak{y}(u, v)+\delta z(u, v) ;(u, v) \in \bar{P}\}
$$

and set $\ell(\epsilon, \delta)=\int_{-1}^{+1}\left|\overline{\mathfrak{r}}_{u}(u, 0 ; \epsilon, \delta)\right| d u$ and $d(\epsilon, \delta)=D[\overline{\mathfrak{r}}]$. A careful

${ }^{2}$ In a forthcoming paper [7] which has been seen by the author as a preprint. S. Hildebrandt proves that the vector $\mathfrak{r}(u, v)$ belongs to class $C^{0,1 / 2}\left(\bar{P}_{r}\right)$. 
analysis shows that, despite the possibly existing zeros of $\left|\mathfrak{r}_{u}(u, 0)\right|$,

$$
\begin{aligned}
\ell(\epsilon, \delta)= & \ell_{2}+\epsilon \int_{-1}^{+1} \frac{\mathfrak{r}_{u}(u, 0) \mathfrak{y}_{u}(u, 0)}{\left|\mathfrak{r}_{u}(u, 0)\right|} d u \\
& +\delta \int_{-1}^{+1} \frac{\mathfrak{r}_{u}(u, 0)_{z_{u}}(u, 0)}{\left|\mathfrak{r}_{u}(u, 0)\right|} d u+o\left(\left(\epsilon^{2}+\delta^{2}\right)^{1 / 2}\right), \\
d(\epsilon, \delta)= & D[\mathfrak{r}]-\epsilon \int_{-1}^{+1} \mathfrak{r}^{*}(u, 0) \mathfrak{y}_{u}(u, 0) d u \\
& -\delta \int_{-1}^{+1} \mathfrak{r}^{*}(u, 0)_{\mho_{u}}(u, 0) d u+O\left(\epsilon^{2}+\delta^{2}\right) .
\end{aligned}
$$

Having established the differentiability of the functions $\ell(\epsilon, \delta)$ and $d(\epsilon, \delta)$ and realizing that the vector $z(u, v)$ can be chosen in such a way that $\partial \ell(0,0) / \partial \delta \neq 0$, the classical derivation of the multiplier rule can be employed (see e.g. [1, pp. 457-465, 542-553, 671]). Since $d(\epsilon, \delta)$ must have a stationary value for $\epsilon=\delta=0$ subject to the subsidiary condition $\ell(\epsilon, \delta)=\ell_{2}$, we conclude that

$$
\int_{-1}^{+1}\left[\mathfrak{r}^{*}(u, 0)+\lambda \frac{\mathfrak{r}_{u}(u, 0)}{\left|\mathfrak{r}_{u}(u, 0)\right|}\right] \mathfrak{y}_{u}(u, 0) d u=0 .
$$

Here $\lambda$ is a constant multiplier. From $\lambda=0$ the impossible relation $\left|\mathfrak{r}_{u}^{*}(u, 0)\right|=\left|\mathfrak{r}_{u}(u, 0)\right|=0$ would follow.

Set $k=1 / \lambda$. Without loss of generality we may assume that $\kappa>0$. As $y(u, v)$ was an arbitrary vector, we find

$$
\frac{\mathfrak{r}_{u}(u, 0)}{\left|\mathfrak{r}_{u}(u, 0)\right|}=-\kappa r^{*}(u, 0)+\mathfrak{c} \quad \text { for almost all } u .
$$

$c$ is a constant vector.

$\partial_{2} P$ is now parametrized by the arc length $s=s(u)=\int_{-1}^{u}\left|\mathfrak{r}_{u}(u, 0)\right| d u$ of $\Gamma_{2} . s(u)$ is a strictly increasing absolutely continuous function. Its inverse function $u=u(s)$ has the same properties.

Setting $\mathfrak{r}^{\circ}(s)=\mathfrak{r}(u(s), 0)$ and $\mathfrak{t}^{\circ}(s)=-\mathfrak{r}^{*}(u(s), 0)+\mathfrak{c}$ it is seen that

$$
\mathfrak{r}^{\circ}(s)=\mathfrak{r}(-1,0)+\int_{-1}^{u(s)} \mathfrak{r}_{u}(u, 0) d u=\mathfrak{r}(-1,0)+\int_{0}^{\circ} \mathfrak{t}^{\circ}(s) d s .
$$

Furthermore, since $\left|\mathfrak{r}_{u}^{*}(u, 0)\right|=\left|\mathfrak{r}_{u}(u, 0)\right|$ almost everywhere,

$$
\left|\mathfrak{t}^{\circ}\left(s_{2}\right)-\mathfrak{t}^{\circ}\left(s_{1}\right)\right|=\left|\int_{u\left(s_{1}\right)}^{u\left(s_{2}\right)} \mathfrak{r}_{u}^{*}(u, 0) d u\right| \leqq \kappa\left|s_{2}-s_{1}\right|
$$


so that the vector $\mathrm{t}^{\circ}(s)$ satisfies a Lipschitz condition. From this it follows that $\Gamma_{2}=\left\{\mathfrak{x}=\mathfrak{x}^{\circ}(s) ; 0 \leqq s \leqq \ell_{2}\right\}$ is a regular curve of class $C^{1,1}$. The results of $[10]$ now tell us that the vector $\mathfrak{x}(u, v)$ belongs to class $C^{1, \alpha}\left(\bar{P}_{r}\right)$ for every $r, 0<r<1$, and every $\alpha, 0<\alpha<1$.

Assume that $\mathfrak{x}_{u}\left(u_{0}, 0\right)=\mathfrak{0}$ for a value $u_{0}$ in $-1<u_{0}<1$. One can convince oneself that the asymptotic expansion, given in $[10, \mathrm{pp}$. $318,329-332]$ and $[5$, p. 103], whose proof is based on a theorem of P. Hartman and A. Wintner [4, Theorems 1, 2 and especially pp. 455-458], can be derived already for a bounding contour of class $C^{1,1}$. Thus, near the point $\left(u_{0}, 0\right)$,

$$
\mathfrak{r}_{u}(u, v)-i \mathfrak{r}_{v}(u, v)=(\mathfrak{a}-i \mathfrak{b})\left(w-w_{0}\right)^{p}+R\left(\left|w-w_{0}\right|\right) .
$$

Here $w=u+i v, w_{0}=u_{0}$. $\mathfrak{a}$ and $\mathfrak{b}$ are nonvanishing real vectors satisfying the relations $\mathfrak{a}^{2}=\mathfrak{b}^{2}, \mathfrak{a} \mathfrak{b}=0$, and $p$ is an integer not less than one. The remainder term $R\left(\left|w-w_{0}\right|\right)$ is of the order $o\left(\left|w-w_{0}\right| p\right)$. A more careful scrutiny of the representation formula of Hartman and Wintner reveals that $R\left(\left|w-w_{0}\right|\right)=O\left(\left|w-w_{0}\right| p+1|\log | w-w_{0}||\right)$. We shall use here that $R\left(\left|w-w_{0}\right|\right)=O\left(\left|w-w_{0}\right| p+\gamma\right)$ for any $\gamma, 0<\gamma<1$.

It is now obvious that the branch points on $\partial_{2} P$ are isolated and that (*) holds for all $u$ in $-1<u<1$. Repeatedly applying the results of [10] it then follows from $(*)$ that $\mathfrak{x}(u, v)$ is of class $C^{\infty}$ in any closed subdomain of $\bar{P}_{r}$ which does not contain the branch points on $\partial_{2} P$. Moreover, from the asymptotic expansion and from the continuity of $\mathrm{t}^{\circ}(s)$ it is seen that the exponent $p$ must be an even number so that the vector $\mathfrak{n}^{\circ}(s)=\mathrm{t}^{\circ \prime}(s) / \kappa=\mathfrak{r}_{v}(u(s), 0) /\left|\mathfrak{r}_{u}(u(s), 0)\right|$ is continuous for $0<s<l_{2}$.

At this point differential geometric considerations have to be brought into play. We set $E(u, v)=\mathfrak{x}_{u}^{2}(u, v)$ and denote by $\mathfrak{X}(u, v)$ the normal vector of $S$ and by $L(u, v), M(u, v), N(u, v)$ the coefficients of the second fundamental form.

A comparison, using (*) and the Gauss equations for the second derivatives of the position vector $\mathfrak{x}(u, v)$, shows that $E_{v}(u, 0)$ $=-2 \kappa E^{3 / 2}(u, 0)$ and $L(u, 0)=0$ in any $u$-interval on $\partial_{2} P$ between branch points. While $\mathfrak{n}^{\circ}(s)$ is the principal normal vector of the curve $\Gamma_{2}$, its binormal vector $\mathfrak{b}^{\circ}(s)$ is given by $\mathfrak{X}(u(s), 0)$ and is also continuous for $0<s<\ell_{2}$. By Frenet's formulas we have $\mathfrak{n}^{\circ \prime}(s)$ $=-\kappa \mathrm{t}^{\circ}(s)+\sigma(s) \mathfrak{b}^{\circ}(s)$. The torsion $\sigma(s)$ of $\Gamma_{2}$ is seen to be equal to the expression $M(u(s), 0) / E(u(s), 0)$.

From the Codazzi equations it follows that $L(u, v)-i M(u, v)$ is in $P$ an analytic function $g(w)$ of the complex variable $w=u+i v$. Since $L(u, 0)=0$ in all points of $\partial_{2} P$ with the exception of isolated points, 
we see that $g(w)$ can be extended as a single valued analytic function in the disc $|w|<1$ punctured at the branch points on $\partial_{2} P$.

Applying the theorem of Gauss-Bonnet, as in [6, p. 233], [9, p. 236], it follows that the total curvature

$$
\iint_{P_{r}}|K| d o=\iint_{P_{r}}|g(w)|^{2} \frac{d u d v}{E(u, v)}
$$

must be finite.

Consider now a branch point on $\partial_{2} P$, say the point $(0,0)$, so that $\mathfrak{r}_{u}(u, v)-i \mathfrak{r}_{v}(u, v)=(\mathfrak{a}-i \mathfrak{b}) w^{p}+O\left(|w|^{p+\gamma}\right)$ near this point. From the finiteness of the total curvature it is seen, that $g(w)$ has no essential singularity at $w=0$ and has an expansion $g(w)=c_{q} w^{q}+c_{q+1} w^{q+1}+\cdots$, $c_{q} \neq 0$, where $q \geqq p$. Thus

$$
\sigma(s) d s=\frac{M(u(s), 0)}{E(u(s), 0)}\left|\mathfrak{r}_{u}(u(s), 0)\right| d u=\left\{\frac{i c_{q}}{|\mathfrak{a}|} u^{q-p}+\cdots\right\} d u,
$$

and the expression in the brackets remains finite. Then, for $0<u_{1}<u_{2}$ and $s_{1}=s\left(u_{1}\right), s_{2}=s\left(u_{2}\right)$,

$$
\begin{aligned}
\mathfrak{r}^{\circ \prime \prime}\left(s_{2}\right)-\mathfrak{r}^{\circ \prime \prime}\left(s_{1}\right) & \\
& =\kappa \int_{s_{1}}^{s_{2}} \mathfrak{n}^{\circ \prime}(s) d s \\
& =-\kappa^{2} \int_{a_{1}}^{s_{2}} \mathfrak{t}^{\circ}(s) d s+\kappa \int_{s_{1}}^{s_{2}} \sigma(s) \mathfrak{b}^{\circ}(s) d s \\
& =-\kappa^{2}\left[\mathfrak{r}^{\circ}\left(s_{2}\right)-\mathfrak{r}^{\circ}\left(s_{1}\right)\right]+\frac{i \kappa c_{q}}{|\mathfrak{a}|} \int_{u_{1}}^{u_{2}} u^{q-p}[1+\cdots] \mathfrak{X}(u, 0) d u,
\end{aligned}
$$

so that $\left|\mathfrak{x}^{\circ \prime \prime}\left(s_{2}\right)-\mathfrak{x}^{\circ \prime \prime}\left(s_{1}\right)\right| \leqq \mathfrak{C}_{1}\left\{\left|s_{2}-s_{1}\right|+\left|u_{2}-u_{1}\right|\right\}$. Using the inequality $\left(u_{2}^{p+1+\gamma}-u_{1}^{p+1+\gamma}\right) /\left(u_{2}^{p+1}-u_{1}^{p+1}\right) \leqq(p+1+\gamma) u_{2}^{\gamma} /(p+1)$, it is seen from the asymptotic expansion that $s_{2}-s_{1} \geqq \mathfrak{C}_{2}\left(u_{2}^{p+1}-u_{1}^{p+1}\right), \mathfrak{C}_{2}>0$, if $u_{2}$ is sufficiently small. From this it follows that $u_{2}-u_{1} \leqq \mathfrak{C}_{3}\left(s_{2}-s_{1}\right)^{1 /(p+1)}$ and therefore $\left|\mathfrak{x}^{\circ \prime \prime}\left(s_{2}\right)-\mathfrak{x}^{\circ \prime \prime}\left(s_{1}\right)\right| \leqq \mathfrak{C}_{4}\left|s_{2}-s_{1}\right|^{1 /(p+1)}$.

It is now possible to conclude that the vector $\mathfrak{x}^{0 \prime \prime}(s)$ satisfies a Hölder condition in every closed subinterval of $0<s<\ell_{2}$, with an exponent depending on the order of the branch points in this subinterval. Therefore, the portion of $\Gamma_{\mathbf{2}}$ corresponding to the interval $|u| \leqq r$ is a curve of class $C^{2, \beta}$ where $\beta=\beta(r)$ is a number depending on $r$. By [10] the vector $\mathfrak{x}(u, v)$ likewise is of class $C^{2, \beta}\left(\bar{P}_{r}\right)$.

Our theorem is proved. The analytic character of the movable 
contours and the absence of branch points on these contours will be the subject of a subsequent investigation.

ACKNOWLEDGEMENT. The preceding research was sponsored by the Air Force Office of Scientific Research under AFOSR Grant No. 883-67.

\section{BIBLIOGRAPHY}

1. O. Bolza, Vorlesungen ïber Variationsrechnung, Teubner, Leipzig and Berlin, 1909.

2. C. V. Boys, Soap bubbles: Their colours and the forces which mold them, Dover, New York, 1959.

3. R. Courant, Soap film experiments with minimal surfaces, Amer. Math. Monthly 47 (1940), 167-174. MR 1, 270.

4. P. Hartman and A. Wintner, On the local behavior of solutions of non-parabolic partial differential equations, Amer. J. Math. 75 (1953), 449-476. MR 15, 318.

5. E. Heinz, Über das Randverhalten quasilinearer elliptischer Systeme mit isothermen Parametern, Math. Z. 113 (1970), 99-105.

6. E. Heinz and S. Hildebrandt, On the number of branch points of surfaces of bounded mean curvature, J. Differential Geometry 4 (1970), 227-235.

7. S. Hildebrandt, Ein einfacher Beweis für die Regularität der Lösungen gewisser zweidimensionaler Variationsprobleme unter freien Randbedingungen, Math. Ann. (to appear).

8. H. Minkowski, Kapillarität, Enc. d. math. Wissensch., 5.1.9, Teubner, Leipzig, 1903-1921, pp. 558-613.

9. J. C. C. Nitsche, On new results in the theory of minimal surfaces, Bull. Amer. Math. Soc. 71 (1965), 195-270. MR 30 \#4200.

10. - The boundary behavior of minimal surfaces. Kellogg's theorem and Branch points on the boundary, Invent. Math. 8 (1969), 313-333. MR 41 \#4399a.

11. - An isoperimetric property of surfaces with moveable boundaries, Amer. Math. Monthly 77 (1970), 359-362. MR 41 \#2547.

12. M. Tsuji, On a theorem of F. and M. Riesz, Proc. Imp. Acad. Tokyo 18 (1942), 172-175. MR 7, 325.

13. K. L. Wolf, Tropfen, Blasen und Lamellen, Springer, Berlin and New York, 1968.

University of Minnesota, Minneapolis, Minnesota 55455 